\section{Asociación predictiva entre parámetros de condición física y dimensiones de calidad de vida relacionada con la salud en adultos mayores chilenos insertos en la comunidad}

\author{
FRANCISCO GUEDE ROJAS ${ }^{1, \mathrm{a},}$, LUIS JAVIER CHIROSA RÍOS ${ }^{2, \mathrm{a}}$, \\ SERGIO FUENTEALBA URRA ${ }^{3, b}$, CÉSAR VERGARA RÍOS ${ }^{1, \mathrm{c}}$, \\ DAVID ULLOA DÍAZ ${ }^{4, a}$, CHRISTIAN CAMPOS JARA ${ }^{1, a}$, \\ PAOLA BARBOSA GONZÁLEZ ${ }^{3, \mathrm{~d}}$, JESUALDO CUEVAS ABURTO ${ }^{4, b}$
}

\section{Association between physical fitness parameters and health related quality of life in Chilean community-dwelling older adults}

Background: There is no conclusive evidence about the association between physical fitness $(P F)$ and health related quality of life (HRQOL) in older adults. Aim: To seek for an association between PF and HRQOL in non-disabled community-dwelling Chilean older adults. Material and Methods: One hundred and sixteen subjects participated in the study. PF was assessed using the Senior Fitness Test (SFT) and hand grip strength (HGS).

HRQOL was assessed using eight dimensions provided by the SF-12v2 questionnaire. Binary multivariate logistic regression models were carried out considering the potential influence of confounder variables. Results: Non-adjusted models, indicated that subjects with better performance in arm curl test (ACT) were more likely to score higher on vitality dimension $(O R>1)$ and those with higher HGS were more likely to score higher on physical functioning, bodily pain, vitality and mental health $(O R>1)$. The adjusted models consistently showed that ACT and HGS predicted a favorable perception of vitality and mental health dimensions respectively $(O R>1)$. Conclusions: HGS and ACT have a predictive value for certain dimensions of HRQOL.

(Rev Med Chile 2017; 145: 55-62)

Key words: Aged; Geriatric Assessment; Physical Fitness; Quality of Life.
${ }^{1}$ Facultad de Ciencias de la Rehabilitación, Universidad Andres Bello, sede Concepción, Chile.

${ }^{2}$ Facultad de Ciencias de la Actividad Física y Deporte, Universidad de Granada, Granada, España.

${ }^{3}$ Facultad de Educación, Universidad Andres Bello, sede Concepción, Chile.

${ }^{4}$ Facultad de Educación, Universidad Católica de la Santísima Concepción, sede Concepción, Chile.

aDoctor en Ciencias de la Actividad Física.

${ }^{\text {b} M a g i ́ s t e r ~ e n ~ E d u c a c i o ́ n . ~}$

'Master of Business

Administration.

${ }^{\mathrm{d}}$ Magíster en Actividad Física y Salud.

Fuente de apoyo financiero: Trabajo financiado por el Fondo Nacional de Investigación en Salud (FONIS), código de proyecto SA12I2229. Se declara que no hubo participación de dicha entidad sobre ningún aspecto de la investigación.

Recibido el 18 de mayo de 2016, aceptado el 29 de diciembre de 2016.

Correspondencia a: Francisco Guede Rojas Autopista 7100, ConcepciónTalcahuano, Chile. Teléfono: (41) 2062302 francisco.guede@unab.cl

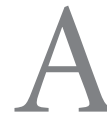

ctualmente las personas son más longevas, situación que ha generado complejos fenómenos sanitarios y sociales ${ }^{1}$. Así, menores tasas de natalidad y mayor esperanza de vida han determinado una transición demográfica hacia el envejecimiento poblacional, lo que, sumado a la creciente carga de enfermedades crónicas y sedentarismo, incrementan la probabilidad de hospitalización e institucionalización de adultos mayores $(\mathrm{AM})^{2}$. Bajo este contexto, la calidad de vida relacionada con la salud (CVRS) y prevención del declive funcional con el envejecimiento, constituyen una prioridad en salud pública, debido a que su comprensión permite el planteamiento de políticas efectivas orientadas al bienestar de la población mayor ${ }^{1,2}$. 
La CVRS, entendida como la percepción de salud física y mental en el tiempo ${ }^{3}$, ha adquirido creciente interés debido a su asociación con diversos resultados objetivos en salud, como el estado nutricional ${ }^{4}$, número de enfermedades crónicas y mortalidad 5 . Por otra parte, la conservación de las capacidades que definen la condición física (CF) como fuerza, flexibilidad, capacidad aeróbica y agilidad/equilibrio dinámico, corresponde a uno de los factores clave para favorecer la independencia funcional y prevención de discapacidad en $\mathrm{AM}^{6,7}$. En Chile, existen antecedentes acerca del comportamiento de ciertos parámetros de $\mathrm{CF}^{8-10}$ y CVRS en población mayor ${ }^{11}$; sin embargo, en el sistema sanitario no se considera la valoración integral de estos aspectos como parte del cuidado general de AM.

De acuerdo a la Organización Mundial de la Salud (OMS), el estado de salud del AM se representa mediante su capacidad para desarrollar las actividades cotidianas y nivel de participación social, reconociendo a la actividad física (AF) como una de las principales estrategias no farmacológicas para favorecer el envejecimiento activo y saludable ${ }^{12}$. Al respecto, si bien la interdependencia entre AF y CVRS es ampliamente reconocida ${ }^{3,13,14}$, la asociación entre CF y CVRS no ha sido suficientemente descrita, considerándose actualmente que la comprensión de los factores predisponentes de la percepción de salud aún es limitado ${ }^{15,16}$.

La literatura ha sugerido la capacidad predictiva de la CF sobre la CVRS en AM autovalentes insertos en la comunidad ${ }^{17-19}$. Sin embargo, no existe consenso acerca de la contribución de cada componente de CF sobre las distintas dimensiones de salud, probablemente debido a que no todas las investigaciones han valorado integralmente la CF mediante baterías estandarizadas. Adicionalmente, se ha planteado que los resultados obtenidos en población mayor deben ser interpretados con cautela, debido a que los diferentes contextos clínicos y metodologías de análisis no han hecho posible la generalización de ellos ${ }^{20-23}$. Por tanto, el objetivo de este trabajo fue identificar el comportamiento predictivo de cada uno de los parámetros esenciales de la CF sobre la CVRS, evaluada multidimensionalmente en una muestra de AM chilenos autovalentes insertos en la comunidad.

\section{Materiales y Métodos}

\section{Diseño y participantes}

En esta investigación transversal se consideró como población de referencia AM pertenecientes al Centro Comunitario de Salud Familiar (CECOSF) "Libertad Gaete" (Talcahuano, Chile). La muestra fue seleccionada mediante un muestreo no probabilístico a partir del cumplimiento de criterios de participación. Los sujetos fueron citados grupalmente a una charla para dar a conocer los objetivos del estudio y luego, en una segunda instancia, se llevó a cabo un chequeo médico y la administración de las pruebas.

Los criterios de inclusión fueron AM "autovalentes" de acuerdo a la Evaluación Funcional del Adulto Mayor (EFAM) ${ }^{24}$, obtención de una puntuación mayor a 13 puntos en el examen Mini-Mental Status Examination (MMSE) abreviado $^{24,25}$ y contar con certificación médica que acredite un estado de salud compatible. Se excluyeron sujetos con ceguera e hipoacusia severa, amputaciones, secuelados de accidente vascular encefálico y presentar cualquier condición que pudiese impedir la realización adecuada de las pruebas. La muestra se conformó por 116 sujetos, quienes firmaron voluntariamente un consentimiento informado. Todos los procedimientos fueron aprobados por el Comité de Bioética de la Universidad Andrés Bello bajo las normas de Helsinki.

\section{Procedimientos}

\section{a) Antropometría}

El peso y talla fueron medidos en una balanza/ tallímetro Detecto ${ }^{\circledR} 2391$ (USA). El índice de masa corporal (IMC) se calculó mediante la ecuación de Quetelet $\left(\mathrm{IMC}=\mathrm{Peso} / \mathrm{Talla}^{2}\right)$ y fue clasificado de acuerdo a los criterios de la OMS para $\mathrm{AM}^{26}$. El perímetro de cintura se midió entre el borde inferior de la última costilla palpable y el borde superior de la cresta iliaca. El perímetro de cadera se midió alrededor de la parte más prominente de las nalgas ${ }^{27}$ utilizando una cinta métrica Ross$\mathrm{craft}^{\circledR}$ (Canadá). El índice cintura/cadera (ICC) se determinó dividiendo el perímetro de cintura por el de cadera y se clasificó de acuerdo a la $\mathrm{OMS}^{27}$.
b) Condición física
Condición física funcional (CFF). Se valoró mediante la batería estandarizada Senior Fitness 
Test (SFT), la cual se conforma por seis pruebas físicas con alta validez y confiabilidad ${ }^{28}$. Las pruebas fueron: 1) Sentarse y levantarse de la silla (SLS): Número de veces que el sujeto logra sentarse y levantarse de una silla durante 30 segundos; 2) Flexión de codo (FDC): Número de flexiones de codo realizadas durante 30 segundos sosteniendo una mancuerna en la mano dominante (3 y 5 libras para mujeres y hombres, respectivamente); 3) Paso 2 min (P2M): Número de veces que el sujeto levanta las rodillas alternadamente hasta el punto medio entre la rótula y la cresta iliaca durante $2 \mathrm{~min}$; 4) Sentarse y alcanzar el pie (SAP): Distancia (centímetros) entre la punta de los dedos de la mano y la punta del pie en posición sedente; 5) Juntar las manos detrás de la espalda (JME): Distancia (centímetros) entre la punta de los dedos de ambas manos; 6) Levantarse, caminar y sentarse (LCS): Tiempo (segundos) que tarda el sujeto en levantarse de una silla, caminar rodeando un cono ubicado a 2,44 metros y volver a sentarse.

Fuerza prensil de mano (FPM). Se midió en sedente con el brazo dominante aducido, el codo flexionado a $90^{\circ}$ y el segmento antebrazo-muñeca neutral, de acuerdo a la Sociedad Americana de Terapeutas de $\mathrm{Mano}^{29}$. Utilizando un dinamómetro hidráulico Jamar ${ }^{\circledR}$ PC5030J1 (USA) en posición II, los sujetos realizaron tres esfuerzos de prensión rápidamente progresivos hasta alcanzar el máximo posible con una pausa de 30 segundos entre cada uno de ellos. Se registró el mayor valor en kilogramos.

\section{c) Calidad de vida relacionada con la salud}

Se utilizó la versión en español del cuestionario SF-12v2 ${ }^{30}$. Las respuestas fueron trasformadas en una escala de 0-100 puntos mediante el software Quality-Metric-Health-Outcomes-Scoring v4.5 $5^{31}$, el cual utiliza un algoritmo con normas referenciales de la población general norteamericana asumiendo una media de $50 \pm 10$. Las dimensiones valoradas fueron: 1) Función física (FF): Grado en que la salud limita las actividades de subir escaleras y caminar más de 1 hora; 2) Rol físico (RF): Grado en que la salud física interfiere en el trabajo y otras actividades diarias; 3 ) Dolor corporal (DC): Intensidad del dolor y su efecto en el trabajo habitual, tanto fuera como dentro del hogar; 4) Salud general (SG): Valoración personal de la salud; 5) Vitalidad (VT): Sentimiento de vitalidad frente al cansancio y agotamiento; 6) Función social
(FS): Grado en que los problemas de salud física o emocional interfieren en la vida habitual; 7) Rol emocional (RE): Grado en que los problemas emocionales interfieren en el trabajo o actividades cotidianas; 8) Salud mental (SM): Sentimiento de tranquilidad, desánimo o tristeza.

\section{Análisis estadístico}

La estadística descriptiva consideró medias, desviaciones estándar y porcentajes. Para la verificación del supuesto de normalidad se utilizó la prueba Shapiro-Wilk y para la homogeneidad de varianzas la prueba $\mathrm{F}$ de Levene. Las asociaciones predictivas se analizaron mediante modelos de regresión logística (MRL) binarios multivariantes, incluyendo los parámetros de CFF, FPM (variables independientes) y de CVRS (variables dependientes). Los puntajes de cada dimensión de CVRS fueron dicotomizados $(\geq 50$ puntos $=$ favorable; $<50$ puntos $=$ desfavorable), tomando los valores más altos como referencia. Se consideraron MRL no-ajustados que incluyeron sólo las variables descritas y MRL ajustados que incorporaron, además, las siguientes variables confundentes: Género (hombre; mujer), rango etario (65-69 años; 70-90 años), IMC e ICC. La bondad de ajuste de los MRL se determinó mediante la prueba Hosmer-Lemeshow y su estadístico ji-cuadrado $\left(\chi^{2}\right)$. El tamaño del efecto de la asociación predictiva entre cada dimensión de CVRS y cada una de las variables dependientes constituyentes de los MRL se determinó mediante el cálculo de Odds Ratios (OR). Todos los análisis se realizaron mediante el software STATA 13.0 considerando un p valor $\leq 0,05$.

\section{Resultados}

Todos los sujetos de estudio completaron las pruebas y no se registraron complicaciones durante las mismas. Las características demográficas de la muestra se exponen en la Tabla 1. El rango etario fue entre 65 y 90 años. El IMC indicó una condición de "sobrepeso" y el ICC sugirió un "riesgo sustancialmente aumentado" de complicaciones metabólicas y cardiovasculares.

Todos los MRL (no-ajustados y ajustados) presentaron bondades de ajuste $\left(\chi^{2}\right)$ significativas ( $\mathrm{p}>0,05)$, lo cual atribuye capacidad explicativa a cada uno de ellos (Tablas 2 y 3 ). 
Tabla 1. Características demográficas de la muestra de estudio

\begin{tabular}{|lccc|}
\hline & $\begin{array}{c}\text { Total } \\
(\mathbf{n = 1 1 6 )}\end{array}$ & $\begin{array}{c}\text { Hombres } \\
(\mathbf{n = 4 7 )}\end{array}$ & $\begin{array}{c}\text { Mujeres } \\
(\mathbf{n}=\mathbf{6 9 )}\end{array}$ \\
\hline Edad (años) & $72,34 \pm 5,80$ & $72,30 \pm 5,66$ & $72,38 \pm 5,94$ \\
\hline Talla $(\mathrm{m})$ & $1,54 \pm 0,09$ & $1,61 \pm 0,07$ & $1,50 \pm 0,07$ \\
\hline Peso $(\mathrm{kg})$ & $73,62 \pm 16,09$ & $76,75 \pm 15,62$ & $71,49 \pm 16,18$ \\
\hline IMC $\left(\mathrm{kg} / \mathrm{m}^{2}\right)$ & $30,84 \pm 5,77$ & $29,46 \pm 5,01$ & $31,80 \pm 6,10$ \\
\hline ICC & $0,94 \pm 0,08$ & $0,99 \pm 0,08$ & $0,93 \pm 0,08$ \\
\hline
\end{tabular}

Datos presentados como medias \pm desviaciones estándar. IMC: Índice de masa corporal; ICC: Índice cintura/cadera.

Tabla 2. Odds ratios (OR) para las dimensiones de CVRS representativas de salud física, de acuerdo a componentes de condición física

\begin{tabular}{|c|c|c|c|c|c|}
\hline \multirow[b]{2}{*}{$\begin{array}{l}\text { Variables } \\
\text { dependientes }\end{array}$} & \multirow[b]{2}{*}{$\begin{array}{c}\text { Variables } \\
\text { independientes }\end{array}$} & \multicolumn{2}{|c|}{ Modelos no-ajustados } & \multicolumn{2}{|c|}{ Modelos ajustados } \\
\hline & & OR & $\begin{array}{l}\text { Bondad } \\
\text { de ajuste }\end{array}$ & OR & $\begin{array}{l}\text { Bondad } \\
\text { de ajuste }\end{array}$ \\
\hline \multirow{7}{*}{$\begin{array}{l}\text { Función física } \\
\text { (FF) }\end{array}$} & SLS & 1,00 & \multirow{7}{*}{$\begin{array}{l}\chi^{2}=7,12 \\
p=0,52\end{array}$} & 1,01 & \multirow{7}{*}{$\begin{aligned} \chi^{2} & =12,98 \\
p & =0,11\end{aligned}$} \\
\hline & FDC & 1,02 & & 1,02 & \\
\hline & P2M & 0,99 & & 0,98 & \\
\hline & SAP & 1,02 & & 1,02 & \\
\hline & JME & 0,99 & & 0,99 & \\
\hline & LCS & 0,97 & & 0,96 & \\
\hline & FPM & $1,07^{*}$ & & 1,06 & \\
\hline \multirow{7}{*}{$\begin{array}{l}\text { Rol físico } \\
\text { (RF) }\end{array}$} & SLS & 1,05 & \multirow{7}{*}{$\begin{array}{c}\chi^{2}=12,21 \\
p=0,14\end{array}$} & 1,06 & \multirow{7}{*}{$\begin{aligned} \chi^{2} & =11,50 \\
p & =0,17\end{aligned}$} \\
\hline & FDC & 0,98 & & 0,99 & \\
\hline & $\mathrm{P} 2 \mathrm{M}$ & 1,01 & & 1,00 & \\
\hline & SAP & 1,03 & & 1,04 & \\
\hline & JME & 1,01 & & 1,01 & \\
\hline & LCS & 1,02 & & 1,01 & \\
\hline & FPM & 1,04 & & 1,03 & \\
\hline \multirow{7}{*}{$\begin{array}{l}\text { Dolor corporal } \\
\text { (DC) }\end{array}$} & SLS & 0,82 & \multirow{7}{*}{$\begin{array}{l}\chi^{2}=6,61 \\
p=0,57\end{array}$} & 0,80 & \multirow{7}{*}{$\begin{aligned} \chi^{2} & =12,14 \\
p & =0,14\end{aligned}$} \\
\hline & FDC & 1,02 & & 1,03 & \\
\hline & P2M & 1,01 & & 1,01 & \\
\hline & SAP & 1,00 & & 1,00 & \\
\hline & JME & 1,00 & & 1,00 & \\
\hline & LCS & 0,91 & & 0,90 & \\
\hline & FPM & $1,05^{*}$ & & 1,05 & \\
\hline \multirow{7}{*}{$\begin{array}{l}\text { Salud general } \\
\text { (SG) }\end{array}$} & SLS & 0,80 & \multirow{7}{*}{$\begin{array}{c}\chi^{2}=7,52 \\
p=0,48\end{array}$} & 0,75 & \multirow{7}{*}{$\begin{array}{l}\chi^{2}=6,73 \\
p=0,56\end{array}$} \\
\hline & FDC & 0,99 & & 1,01 & \\
\hline & P2M & 1,02 & & 1,03 & \\
\hline & SAP & 1,05 & & 1,07 & \\
\hline & JME & 1,01 & & 1,01 & \\
\hline & LCS & 0,72 & & 0,71 & \\
\hline & FPM & 1,05 & & 1,05 & \\
\hline
\end{tabular}

*Asociación predictiva con significación estadística; SLS: Sentarse y levantarse de la silla; FDC: Flexiones de codo; P2M: Paso 2 min; SAP: Sentarse y alcanzar el pie; JME: Juntar las manos detrás de la espalda; LCS: Levantarse, caminar y sentarse; FPM: Fuerza prensil de mano. 
Tabla 3. Odds ratios (OR) para las dimensiones de CVRS representativas de salud mental, de acuerdo a componentes de condición física

\begin{tabular}{|c|c|c|c|c|c|}
\hline $\begin{array}{l}\text { Variables depen- } \\
\text { dientes }\end{array}$ & $\begin{array}{l}\text { Variables indepen- } \\
\text { dientes }\end{array}$ & $\begin{array}{l}\text { Mode } \\
\text { OR }\end{array}$ & $\begin{array}{l}\text {-ajustados } \\
\text { Bondad } \\
\text { de ajuste }\end{array}$ & $\begin{array}{l}\text { Mo } \\
\text { OR }\end{array}$ & $\begin{array}{l}\text { justados } \\
\text { Bondad } \\
\text { de ajuste }\end{array}$ \\
\hline $\begin{array}{l}\text { Vitalidad } \\
\text { (VT) }\end{array}$ & $\begin{array}{l}\text { SLS } \\
\text { FDC } \\
\text { P2M } \\
\text { SAP } \\
\text { JME } \\
\text { LCS } \\
\text { FPM }\end{array}$ & $\begin{array}{l}0,95 \\
1,14^{*} \\
0,98 \\
1,00 \\
1,00 \\
0,96 \\
1,05^{*}\end{array}$ & $\begin{array}{l}\chi^{2}=7,06 \\
p=0,53\end{array}$ & $\begin{array}{l}0,93 \\
1,15^{*} \\
0,98 \\
1,00 \\
1,00 \\
0,96 \\
1,06\end{array}$ & $\begin{array}{l}\chi^{2}=4,49 \\
p=0,81\end{array}$ \\
\hline $\begin{array}{l}\text { Función social } \\
\text { (FS) }\end{array}$ & $\begin{array}{l}\text { SLS } \\
\text { FDC } \\
\text { P2M } \\
\text { SAP } \\
\text { JME } \\
\text { LCS } \\
\text { FPM }\end{array}$ & $\begin{array}{l}0,95 \\
1,05 \\
1,00 \\
1,00 \\
1,00 \\
1,01 \\
1,01\end{array}$ & $\begin{array}{c}\chi^{2}=13,43 \\
p=0,09\end{array}$ & $\begin{array}{l}0,96 \\
1,06 \\
1,00 \\
1,01 \\
1,00 \\
1,00 \\
0,99\end{array}$ & $\begin{array}{l}\chi^{2}=9,03 \\
p=0,33\end{array}$ \\
\hline $\begin{array}{l}\text { Rol emocional } \\
\text { (RE) }\end{array}$ & $\begin{array}{l}\text { SLS } \\
\text { FDC } \\
\text { P2M } \\
\text { SAP } \\
\text { JME } \\
\text { LCS } \\
\text { FPM }\end{array}$ & $\begin{array}{l}0,95 \\
1,06 \\
1,00 \\
0,98 \\
0,99 \\
0,97 \\
1,03\end{array}$ & $\begin{array}{l}\chi^{2}=3,46 \\
p=0,90\end{array}$ & $\begin{array}{l}0,93 \\
1,07 \\
1,00 \\
0,98 \\
0,99 \\
0,95 \\
1,05\end{array}$ & $\begin{array}{l}\chi^{2}=8,10 \\
p=0,42\end{array}$ \\
\hline $\begin{array}{l}\text { Salud mental } \\
\text { (SM) }\end{array}$ & $\begin{array}{l}\text { SLS } \\
\text { FDC } \\
\text { P2M } \\
\text { SAP } \\
\text { JME } \\
\text { LCS } \\
\text { FPM }\end{array}$ & $\begin{array}{l}0,78 \\
1,06 \\
0,99 \\
0,95 \\
1,01 \\
0,86 \\
1,05^{*}\end{array}$ & $\begin{array}{c}\chi^{2}=12,17 \\
p=0,14\end{array}$ & $\begin{array}{l}0,77 \\
1,06 \\
0,99 \\
0,94 \\
1,01 \\
0,85 \\
1,06^{*}\end{array}$ & $\begin{array}{c}\chi^{2}=14,05 \\
p=0,08\end{array}$ \\
\hline
\end{tabular}

*Asociación predictiva con significación estadística; SLS: Sentarse y levantarse de la silla; FDC: Flexiones de codo; P2M: Paso 2 min; SAP: Sentarse y alcanzar el pie; JME: Juntar las manos detrás de la espalda; LCS: Levantarse, caminar y sentarse; FPM: Fuerza prensil de mano.

La Tabla 2, en particular, muestra las asociaciones predictivas para las dimensiones de CVRS representativas de salud física (FF, RF, DC y SG). Bajo los modelos no-ajustados, los sujetos que presentaron mayor FPM fueron más propensos a valorar favorablemente las dimensiones FF y DC ( $\mathrm{OR}=1,07$ y 1,05 respectivamente), lo cual no se observó bajo los respectivos modelos ajustados. Por su parte, la Tabla 3 muestra las asociaciones predictivas para las dimensiones de CVRS representativas de salud mental (VT, FS, RE y SM). Los modelos no-ajustados muestran que sujetos con mayor FPM fueron más propensos a presentar una percepción positiva de las dimensiones VT y SM $(\mathrm{OR}=1,05)$. Además, aquellos con mejor rendimiento en la prueba FDC percibieron positivamente la dimensión VT $(\mathrm{OR}=1,14)$. Los modelos ajustados indicaron consistentemente que aquellos con mejores resultados en las pruebas FDC y FPM presentaron una mayor probabilidad de valorar favorablemente las dimensiones VT y $\mathrm{SM}(\mathrm{OR}=1,15$ y 1,06 respectivamente $)$. 


\section{Discusión}

A la fecha, las asociaciones entre CF y CVRS no han sido suficientemente descritas especialmente en $\mathrm{AM}^{15,16}$. Por tanto, la presente investigación indagó en el comportamiento predictivo de diferentes parámetros de CF sobre la CVRS valorada multidimensionalmente, tomando en cuenta la influencia de variables confundentes como la edad $^{32,33}$, género ${ }^{32}, \mathrm{IMC}^{34}$ e ICC ${ }^{35}$.

El principal hallazgo de esta investigación fue que parámetros de CF representativos de la fuerza muscular de extremidad superior, predijeron de mejor manera una positiva valoración de ciertas dimensiones de CVRS, sugiriendo su influencia sobre la percepción de salud física y mental. Los OR oscilaron entre 1,05 y 1,15 , representando el tamaño del efecto relativo a la probabilidad de obtener puntuaciones más altas en las dimensiones de CVRS de acuerdo al desempeño en las pruebas de fuerza. Interesantemente, los MRL ajustados mostraron que las asociaciones entre FDC y FPM con VT y SM respectivamente, serían independientes de la influencia de las variables confundentes, mientras que las asociaciones entre FPM y las dimensiones FF, DC y VT, al ser significativas sólo en los MRL no-ajustados pudieron ser influenciadas por ellas.

Estudios previos concuerdan en la capacidad predictiva de diversos componentes de la CF sobre la percepción de salud en AM, sugiriendo su relevancia para el diseño efectivo de políticas públicas y programas sanitarios que se proponen como objetivo mejorar la calidad de vida de la población mayor ${ }^{1,2}$. Sin embargo, la heterogeneidad de las investigaciones relativa a las características sociodemográficas poblacionales, pruebas evaluativas, covariables confundentes y metodologías consideradas, complejiza la comparación de resultados y la obtención de conclusiones definitivas $^{15,36}$. En este sentido, Mulasso et $\mathrm{al}^{37}$ reportaron la influencia de características individuales como la edad, condiciones de vida, nivel de educación, historial laboral, farmacoterapia y características antropométricas sobre la CVRS en AM autovalentes insertos en la comunidad.

Los resultados obtenidos concuerdan parcialmente con los reportados en estudios previos que han utilizado metodologías similares. Wanderley et $\mathrm{al}^{19}$, encontraron que AM autovalentes con mayor FPM eran más propensos a presentar mejores resultados en las dimensiones RF y VT. Además, aquellos con mayor capacidad aeróbica puntuaban mejor en las dimensiones FF, RF y VT. Los autores, al igual que en el presente estudio, no encontraron asociaciones significativas entre ningún parámetro de CF y las dimensiones SG, FS y RE. En contraste, Maia et $\mathrm{al}^{38}$ no encontraron asociaciones entre parámetros de CFF y CVRS en AM autovalentes, sin embargo, los autores no incorporaron pruebas de fuerza de extremidad superior, lo cual apoya la importancia de los resultados y aportes del presente estudio. Por otra parte, Brovold et $\mathrm{al}^{21}$ reportaron en AM recientemente dados de alta hospitalaria, que todos los parámetros de CFF predijeron favorablemente las ocho dimensiones de CVRS, sosteniendo que el nivel de AF de la muestra pudo ser un factor determinante para las asociaciones observadas.

Investigaciones han descrito las asociaciones entre CF y la percepción de problemas de salud, evaluada mediante diversos instrumentos como el cuestionario EQ-5D ${ }^{18,23}$ y WHOQOL ${ }^{22,38}$. En esta investigación se utilizó el cuestionario SF-12v2 debido a su corto tiempo de aplicación, validez-confiabilidad ${ }^{39} \mathrm{y}$ a su capacidad de aportar las mismas dimensiones del ampliamente utilizado SF- $36^{40}$.

Dentro de las limitaciones del presente trabajo, se plantea que el diseño transversal utilizado dificulta la posibilidad de evaluar la dirección de causalidad y que el muestreo no probabilístico permite concluir sólo sobre los sujetos evaluados. Además, la falta de control mediante otras variables potencialmente confundentes como $\mathrm{AF}$, estrato socioeconómico y enfermedades crónicas, no permite determinar su posible influencia sobre los resultados. Dentro de las fortalezas del trabajo, se considera la inclusión de una muestra dirigida perteneciente a una población de difícil acceso debido a sus características sociales y la utilización de pruebas e instrumentos válidos para la evaluación de la CF y CVRS en AM. Adicionalmente, si bien la $2^{\circ}$ Encuesta de Calidad de Vida y Salud (ENCAVI) del Ministerio de Salud ${ }^{11}$, entre otros aspectos, ofrece una detallada descripción de todas las dimensiones del cuestionario SF-12v2 de acuerdo a género, edad y estrato socioeconómico, no incluye aspectos relativos a la CF de la población. Por otra parte, estudios nacionales que evalúan el comportamiento de la capacidad aeróbica ${ }^{8}$, $\mathrm{FPM}^{9}$ y equilibrio estático-dinámico ${ }^{10}$ de $\mathrm{AM}$, no han considerado la CVRS. Por tanto, de acuerdo 
a la información disponible por los autores, este es el primer trabajo que integra ambos aspectos, aportando antecedentes acerca de la capacidad predictiva de la CF sobre la CVRS en una muestra de AM chilenos.

En conclusión, los resultados de esta investigación demuestran que la FPM se asocia a una mejor percepción de FF y DC como indicadores de salud física y a una mejor percepción de VT y SM como indicadores de salud mental. Además, un mejor rendimiento en la prueba FDC se asocia a una mejor percepción de VT como indicador de salud mental. Estos resultados revelan que, tanto la prueba FPM como FDC, valoradas según los estándares explicitados en este estudio, poseen un valor predictivo interesante para ciertas dimensiones de CVRS, y que, por tanto, considerando su bajo costo y rápida administración, sería razonable considerar su implementación tanto en programas de atención primaria como en el EFAM ${ }^{24}$.

Agradecimientos: Los autores agradecen a FONIS, a la Dirección de Administración en Salud (DAS) de la Ilustre Municipalidad de Talcahuano y al equipo profesional del CECOSF "Libertad Gaete" por el apoyo permanente durante el desarrollo de la presente investigación.

\section{Referencias}

1. Chatterji S, Byles J, Cutler D, Seeman T, Verdes E. Health, functioning, and disability in older adults. Present status and future implications. Lancet 2015; 385 (9967): 563-75.

2. Kohl H, Craig C, Lambert E, Inoue S, Alkandari J, Leetongin $\mathrm{G}$, et al. The pandemic of physical inactivity: global action for public health. Lancet 2012; 380 (9838): 294-305.

3. Kelley G, Kelley K, Hootman J, Jones D. Exercise and health-related quality of life in older community-dwelling adults a meta-analysis of randomized controlled trials. J Appl Gerontol 2009; 28 (3): 369-94.

4. Yan L, Daviglus M, Liu K, Pirzada A, Garside D, Schiffer L, et al. BMI and Health related quality of life in adults 65 years and older. Obes Res 2004; 12 (1): 69-76.

5. Dominick K, Ahern F, Gold C, Heller D. Relationship of health-related quality of life to health care utilization and mortality among older adults. Aging Clin Exp Res 2002; 14 (6): 499-508.

6. den Ouden M, Schuurmans M, Brand J, Arts I, Mueller-Schotte S, van der Schouw Y. Physical functioning is related to both an impaired physical ability and ADL disability: a ten year follow-up study in middle-aged and older persons. Maturitas 2013; 74 (1): 89-94.

7. de Melo L, Menec V, Ready A. Relationship of Functional Fitness With Daily Steps in Community-Dwelling Older Adults. J Geriatr Phys Ther 2014; 37 (3): 116-20.

8. Díaz J, Espinoza O, Rodríguez H, Moreno A. Prevalencia de patrones antropométricos y fisiológicos en población de adultos mayores, sobre los 60 años en Arica, Chile. Int J Morphol 2011; 29 (4): 1449-54.

9. Mancilla E, Ramos S, Morales P. Fuerza de prensión manual según edad, género y condición funcional en adultos mayores Chilenos entre 60 y 91 años. Rev Med Chile 2016; 144 (5): 598-603.

10. Mancilla E, Valenzuela J, Escobar M. Rendimiento en las pruebas "Timed Up and Go" y "Estación Unipodal” en adultos mayores chilenos entre 60 y 89 años. Rev Med Chile 2015; 143 (1): 39-46.

11. Ministerio de Salud (MINSAL). II Encuesta de calidad de vida (ENCAVI). Informe de resultados total nacional. Disponible en: http://www.crececontigo.gob.cl/wp-content/uploads/2013/06/ENCAVI-2006.pdf [Consultado el 31 de octubre de 2016].

12. Organización Mundial de la Salud (OMS). Recomendaciones mundiales sobre actividad física para la salud. Disponible en: http://www.who.int/dietphysicalactivity/ factsheet_recommendations/es/ [Consultado el 30 de octubre de 2016].

13. Bize R, Johnson J, Plotnikoff R. Physical activity level and health-related quality of life in the general adult population: a systematic review. Prev Med 2007; 45 (6): 401-15.

14. Sun F, Norman I, While A. Physical activity in older people: a systematic review. BMC Public Health 2013; 13 (1): 449-65.

15. Bailey S, Bailey E, Mushti S, McHugh H, Senbarger J. Predictors of Health Related Quality of Life in Adults 50 Years and Older. J Nov Physiother 2016; 6 (1): 1-7.

16. Chung P, Zhao Y, Liu J, Quach B. A canonical correlation analysis on the relationship between functional fitness and health-related quality of life in older adults. Arch Gerontol Geriatr 2016; 68: 44-8.

17. Hörder H, Skoog I, Frändin K. Health-related quality of life in relation to walking habits and fitness: a population-based study of 75-year-olds. Qual Life Res 2013; 22 (6): 1213-23.

18. Olivares P, Gusi N, Prieto J, Hernández-Mocholi M. Fitness and health-related quality of life dimensions in community-dwelling middle aged and older adults. Health Qual Life Outcomes 2011; 9: 117-27.

19. Wanderley F, Silva G, Marques E, Oliveira J, Mota J, Carvalho J. Associations between objectively assessed 
physical activity levels and fitness and self-reported health-related quality of life in community-dwelling older adults. Qual Life Res 2011; 20 (9): 1371-78.

20. Benton M, Alexander J, Holland J. Relationship between strength, function, and quality of life in older adults with chronic lung disease: is there an influence of gender? J Cardiopulm Rehabil Prev 2014; 34 (2): 143-49.

21. Brovold T, Skelton D, Sylliaas H, Mowe M, Bergland A. Association between health-related quality of life, physical fitness, and physical activity in older adults recently discharged from hospital. J Aging Phys Act 2014; 22 (3): 405-13.

22. Cavalcante P, Doro M, Suzuki F, Rica R, Serra A, Pontes Junior F, et al. Functional Fitness and Self-Reported Quality of Life of Older Women Diagnosed with Knee Osteoarthrosis: A Cross-Sectional Case Control Study. J Aging Res 2015; 501: 1-7.

23. Pedrero-Chamizo R, Gómez-Cabello A, Mélendez A, Vila-Maldonado S, Espino L, Gusi N, et al. Higher levels of physical fitness are associated with a reduced risk of suffering sarcopenic obesity and better perceived health among the elderly. The EXERNET multi-center study. J Nutr Health Aging 2015; 19 (2): 211-7.

24. Ministerio de Salud (MINSAL). Manual de Aplicación del Examen de Medicina Preventiva del Adulto Mayor. Disponible en: http://web.minsal.cl/portal/url/item/ ab1f81f43ef0c2a6e04001011e011907.pdf [Consultado el 31 de octubre de 2016].

25. Organización Panamericana de la Salud/Organización Mundial de la Salud (OPS/OMS). Encuesta multicéntrica Salud Bienestar y Envejecimiento (SABE) en América Latina y el Caribe. Washington; 2001. Disponible en: http://www.ssc.wisc.edu/sabe/docs/informeFinal\%20 EspaNol\%20noviembre\%202004.pdf [Consultado el 01 de noviembre de 2016].

26. Organización Panamericana de la Salud/Organización Mundial de la Salud (OPS/OMS). Guía clínica para atención primaria a las personas adultas mayores. Disponible en: http://www.sld.cu/galerias/pdf/sitios/gericuba/introduccion.pdf [Consultado el 01 de noviembre de 2016].

27. World Health Organization (WHO). Waist circumference and waist-hip ratio: Report of a WHO Expert Consultation Disponible en: http://apps.who.int/iris/ bitstream/10665/44583/1/9789241501491_eng.pdf [Consultado el 02 de noviembre de 2016].

28. Rikli R, Jones C. Senior fitness test manual. Canada: Human Kinetics; 2013.
29. Fess E: Grip strength. In Clinical assessment recommendations. 2nd edition. Edited by Casanova JS. Chicago: American Society of Hand Therapists; 1992: 41-5.

30. QualityMetric. Adaptación al español del cuestionario de salud SF-12v2-Chile (Español)-4 semanas. Disponible en: http://www.bibliopro.org/buscador/618/ cuestionario-de-salud-sf-12v2-chile-espanol-4-semanas [Consultado el 02 de noviembre de 2016].

31. Saris-Baglama R, Dewey C, Chisholm G, Plumb E, Kosinski M, Bjorner J, et al. QualityMetric Health Outcomes Scoring Software 2.0: User's Guide. Lincoln, RI: Quality Metric Incorporated; 2007.

32. Rikli R, Jones C. Functional fitness normative scores for community-residing older adults, ages 60-94. J Aging Phys Act 1999; 7: 162-81.

33. Trombetti A, Reid K, Hars M, Herrmann F, Pasha E, Phillips E, et al. Age-associated declines in muscle mass, strength, power, and physical performance: impact on fear of falling and quality of life. Osteoporos Int 2015; 1-9.

34. Cohen A, Baker J, Ardern C. Association Between Body Mass Index, Physical Activity, and Health-Related Quality of Life in Canadian Adults. J Aging Phys Act 2016; 24 (2): 32-8.

35. Daniele T, Bruin V, Oliveira D, Pompeu C. Associations among physical activity, comorbidities, depressive symptoms and health-related quality of life in type 2 diabetes. Arq Bras Endocrinol Metabol 2013; 57 (1): 44-50.

36. Sartor-Glittenberg C, Lehmann S, Okada M, Rosen D, Brewer K, Bay R. Variables explaining health-related quality of life in community-dwelling older adults. J Geriatr Phys Ther 2014; 37 (2): 83-91.

37. Mulasso A, Roppolo M, Rabaglietti E. The role of individual characteristics and physical frailty on health related quality of life (HRQOL): A cross sectional study of Italian community-dwelling older adults. Arch Gerontol Geriatr 2014; 59 (3): 542-48.

38. Maia T, Junior E, Louro J, Vieira E, Gurgel J. Physical Fitness and quality of life of the elderly: a transversal study. Online Braz J Nurs 2014; 13 (4): 559-67.

39. Cheak-Zamora N, Wyrwich K, McBride T. Reliability and validity of the SF-12v2 in the medical expenditure panel survey. Qual Life Res 2009; 18 (6): 727-35.

40. Ware J, Kosinski M, Turner-Bowker D, Gandek B. How to score version 2 of the SF-12 health survey (with a supplement documenting version 1): QualityMetric Incorporated; 2002. 